\title{
Porocarcinoma: reporte de caso y revisión de la literatura
}

\author{
Eduardo Téllez-Bernal ${ }^{1,2 *}$, Francisco Javier Gil-Beristain ${ }^{3}$, María de Jesús Valdés ${ }^{4}$, \\ Jorge Alberto Jiménez-López ${ }^{5}$, Marcela Hernández-Charolet ${ }^{6}$, Miguel Ángel García-Martínez, \\ Edith Téllez-Herrera ${ }^{8}$, Begoña Macías-Alonso ${ }^{9}$ \\ ${ }^{1}$ Servicio de Oncología, ISSSTEP; ${ }^{2}$ Unidad Médica Oncológica; ${ }^{3}$ Servicio de Dermatología, Médica Dermatológica; ${ }^{4}$ Servicio de Patología, ISSSTEP; \\ ${ }^{5}$ Servicio de Oncología Quirúrgica, ISSSTEP; ${ }^{6}$ Supervisión General Médica, ISSSTEP; ${ }^{7}$ Servicio de Oncología Hematológica, ISSSTEP; ${ }^{8}$ Servicio \\ de Investigación, Unidad Médica Oncológica; ${ }^{9}$ Servicio de Medicina Interna, ISSSTEP. Puebla, México.
}

\section{Resumen}

Introducción: El porocarcinoma es una neoplasia dermatológica extraña que se desarrolla en las glándulas sudoríparas de la porción ductal intradérmica. Presentación del caso: Describimos el caso de un paciente masculino de 65 años de edad, mexicano, con una larga historia clínica de lesión en la piel, que fue exitosamente tratada con cirugía y tuvo un intervalo libre de recurrencia de tres años. Discusión: Este tumor representa del 0.005 al $0.01 \%$ de los tumores dermatológicos y es el más frecuente de los tumores ecrinos malignos. Afecta principalmente a las personas que se encuentran entre la quinta y la octava década de la vida; las áreas más afectadas son los miembros inferiores. El tratamiento de elección aún es la resección amplia mediante cirugía micrográfica de Mohs, cuya tasa de curación se encuentra entre el 70 y el $80 \%$. Conclusiones: Aunque la tasa de incidencia del porocarcinoma es muy baja, el comportamiento biológico de este tumor se vuelve agresivo cuando la enfermedad se disemina. Pese a su baja frecuencia, diagnosticarlo aún es un reto.

Palabras clave: Porocarcinoma. Ecrino. Neoplasia. Cáncer dermatológico. Cirugía Mohs

\section{Porocarcinoma: case report and literature review}

\section{Abstract}

Introduction: Porocarcinoma is a strange neoplasm of the skin, which develops in the sweat glands at the intradermal ductal portion. Case Report: We describe the case of a 65-year-old Mexican patient with a long clinical history of skin lesion, which was successfully treated with surgical treatment, and a 3-year recurrence-free interval. Discussion: This tumor represents from $0.005 \%$ to $0.01 \%$ of skin tumors and is the most frequent of the eccrine malignant tumors. It predominantly affects people in the fifth and eighth decade of life; the most commonly affected areas are the lower limbs. The treatment of choice is still the wide surgical resection with Mohs micrographic surgery, whose cure rate ranges from $70 \%$ to $80 \%$. Conclusions: Although porocarcinoma incidence rate is very low, its biological behavior turns it aggressive when the disease spreads. In spite of its low frequency, it is still a diagnose challenge.

Key words: Porocarcinoma. Eccrine. Neoplasm. Skin cancer. Mohs surgery.

\section{Correspondencia:}

E. Téllez Bernal

E-mail: umoetb@yahoo.com
Fecha de recepción: 08-11-2017

Fecha de aceptación: 14-02-2018

DOI: 10.24875/j.gamo.M18000141
Disponible en internet: 13-05-2019 Gac Mex Oncol. 2019;Supp 18:80-83 www.gamo-smeo.com 1665-9201/@ 2018 Sociedad Mexicana de Oncología. Publicado por Permanyer México SA de CV. Este es un artículo Open Access bajo la licencia CC BY-NC-ND (http://creativecommons.org/licenses/by-nc-nd/4.0/). 


\section{Introducción}

El porocarcinoma ecrino es una neoplasia rara de la piel, que se desarrolla en las glándulas sudoríparas en la porción ductal intradérmica ${ }^{1}$. Fue descrita por primera vez por Pinkus y Mehregan ${ }^{2,3}$ en 1963. En 1969, Mishima y Morioka ${ }^{4}$ introdujeron el término actual «porocarcinoma» ${ }^{4}$.

Este tumor representa del 0.005 al $0.01 \%$ de los tumores cutáneos y es la forma más frecuente de los tumores malignos de las glándulas sudoríparas ${ }^{2}$. Debido a su baja incidencia, el comportamiento biológico de la neoplasia no es totalmente conocido, por lo que es importante un adecuado diagnóstico y tratamiento óptimo.

Se ha reportado que aproximadamente el $20 \%$ de los casos recurre después de ser extirpado y puede provocar metástasis a ganglios linfáticos en un $20 \%$ de los casos, así como metástasis a distancia hasta en un $10 \%$; rara vez se disemina a nivel visceral ${ }^{5}$.

Aunque la etiología sigue siendo desconocida, se han descrito como posibles causas la radioterapia, el daño solar y la inmunosupresión; pero no hay clara evidencia que lo sustente.

Los sitios más comúnmente afectados son las extremidades inferiores $(55 \%)$, la cabeza y el cuero cabelludo $(20 \%)$, las extremidades superiores $(20 \%)$ y el tronco y abdomen $(10 \%)^{6}$. Sin embargo, no se ha relacionado con áreas de mayor concentración de las glándulas sudoríparas ecrinas.

El porocarcinoma ecrino afecta a hombres y mujeres por igual, particularmente entre la quinta y la octava década de la vida, aunque se han reportado casos en adultos jóvenes ${ }^{2}$. Usualmente, el tumor presenta larga evolución y podría originarse de un poroma ecrino que maligniza a través de los años. Dicha transformación a una variante maligna es rápida (comúnmente de algunos meses) y ya ha sido documentada.

\section{Presentación del caso}

En el Hospital ISSSTEP (Instituto de Seguridad y Servicio Social de los Trabajadores al Servicio de los Poderes del Estado de Puebla) la frecuencia de tumores de piel es muy alta. En 2013, cuando se encontró este caso, los tumores malignos de piel fueron la primera causa de cáncer, seguidos del cáncer de mama y el de próstata ${ }^{7}$.

\section{Características clínicas del paciente}

Paciente masculino de 65 años de edad con antecedentes familiares de diabetes mellitus tipo 2 e hipertensión arterial sistémica. Originario de la comunidad de
Chiautla de Tapia, Puebla, y residente de Axochiapan, Puebla. Maestro jubilado, actualmente es empacador de especias. Fue campesino durante 13 años (desde los cinco años hasta los 18) y no usó medidas de fotoprotección. Alimentación equilibrada e ingesta adecuada de líquidos. Tabaquismo desde los 16 años, a razón de dos cigarrillos por día (índice tabáquico de 4.2 paquetes-año) e ingesta de bebidas alcohólicas desde los 18 años (durante nueve años con ingesta de cerveza dos veces por semana hasta llegar a la embriaguez). Exposición a humo de leña durante 22 años, a razón de 30 minutos al día (índice de exposición 11 horas/año).

Antecedente de diabetes mellitus tipo 2 desde hace 11 años de diagnóstico, en tratamiento y control. Hipertrigliceridemia e hiperuricemia diagnosticadas hace seis meses, en tratamiento y descontroladas.

Paciente que desde los 19 años de edad presenta dermatosis que afecta mejilla derecha y está constituida por una neoformación exofítica, hipercrómica de color marrón, que medía aproximadamente $2 \mathrm{~cm}$ de diámetro, de bordes precisos y evolución aparentemente crónica. A partir del año 2013, la lesión aumenta progresivamente de tamaño, sin cambios en la coloración y sin sintomatología acompañada. Acude a consulta con facultativo, quien realiza resección de la lesión, la cual es enviada al Servicio de Patología de nuestro hospital, que la describe con las siguientes características:

- Descripción macroscópica. Un huso de piel que mide $2.6 \times 0.5 \mathrm{~cm}$. Rugosa, de color café oscura, con un aumento de volumen en la zona central que mide $0.8 \mathrm{~cm}$; rugoso de color gris, al corte es sólido, de color blanquecino. Se incluyen cortes representativos en una cápsula.

- Descripción microscópica. Los cortes histológicos examinados muestran la piel que presenta la epidermis sin alteraciones (Fig. 1); subyacente se observa tumor maligno de anexos cutáneos con patrón de crecimiento sólido, con proliferación de células de aspecto basaloide con núcleos vesiculosos. Pleomorfismo moderado y un índice mitósico de 4 xCSF (40x) (Fig. 2). La neoplasia presenta formación de lúmenes intracitoplásmicos multifocales (Fig. 3). Los bordes del tumor son infiltrativos a nivel de dermis reticular (Fig. 4). No se identifica permeación vascular linfática. El lecho quirúrgico y bordes quirúrgicos no presentan lesión neoplásica.

\section{Discusión}

En el porocarcinoma ecrino, las lesiones pueden aparecer como un nódulo o una neoformación verrucosa, 


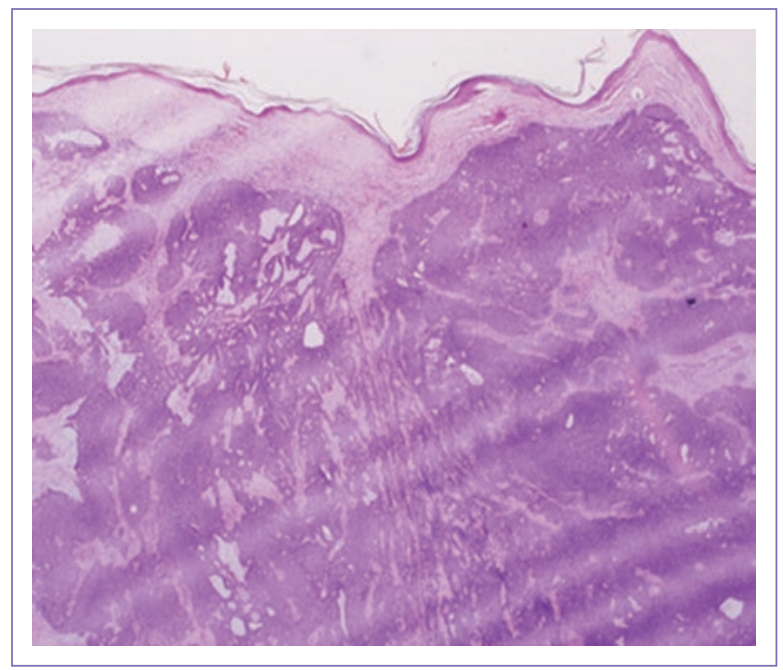

Figura 1. Resección de neoformación de mejilla (magnificación 4x). Neoplasia sólida de epitelio de glándula sudorípara de bordes empujantes.

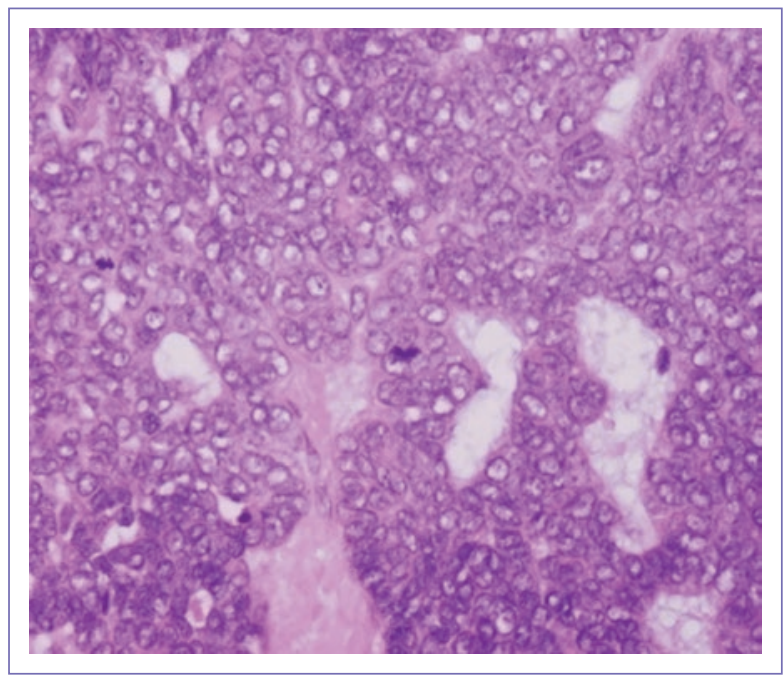

Figura 2. Núcleos vesiculosos con pleomorfismo moderado y algunas mitosis atípicas (amplificación 40x).

en forma de domo, infiltrativo, como placa erosiva o también de crecimiento polipoideo que es frecuentemente ulcerado. En algunos casos pueden asociarse lesiones multinodulares ulcerativas de rápido crecimiento, ya sea con recurrencia local o con metástasis. El promedio de tamaño de las lesiones diagnosticadas es de $2.4 \mathrm{~cm}$ con un rango de $1 \mathrm{a} 10 \mathrm{~cm}^{8}$.

La variante in situ es reconocida por la presencia de células de poroma, con formación de luces, asociada con características citológicas de malignidad.

Los porocarcinomas malignos ecrinos muestran continuidad con la superficie epitelial y pueden estar asociados con bordes profundos infiltrantes. Este tumor

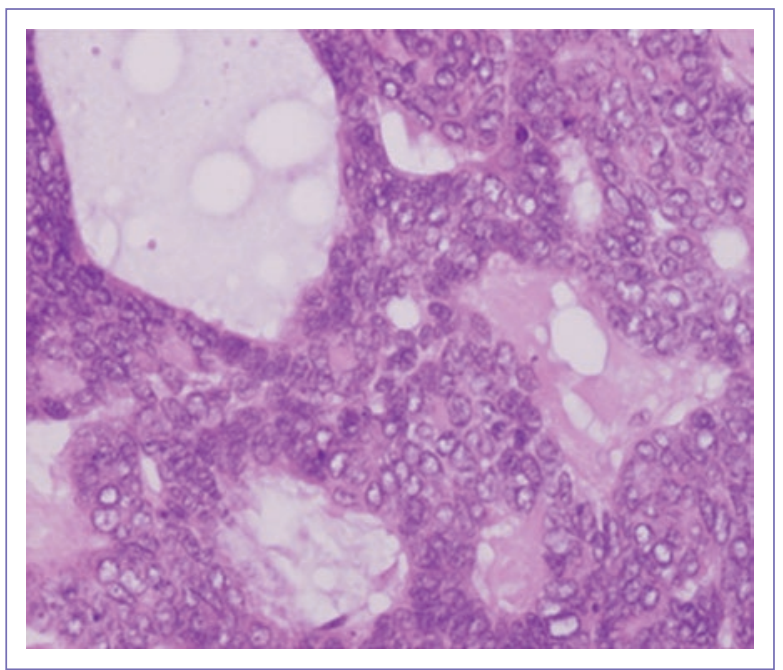

Figura 3. Formación de lúmenes intracitoplásmicos (amplificación 40x).

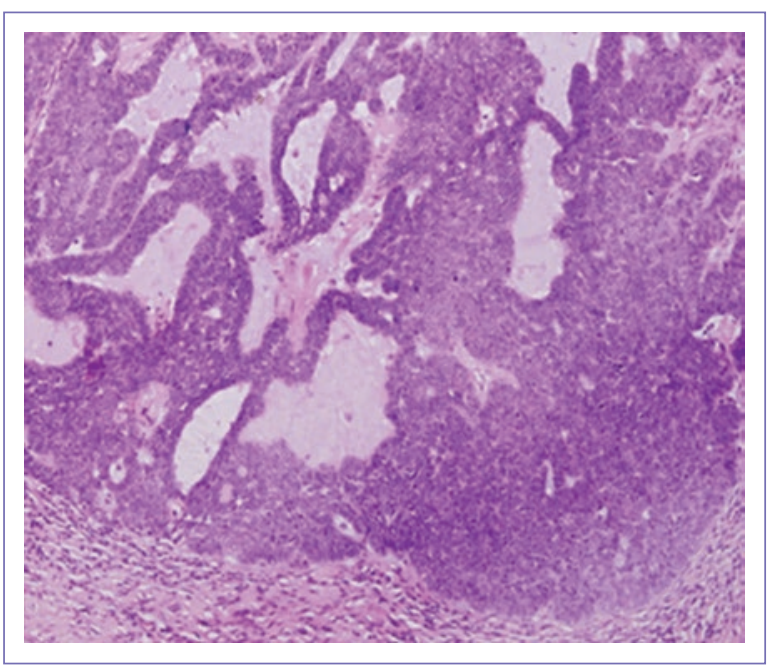

Figura 4. Bordes infiltrativos, leve infiltrado inflamatorio por leucocitos mononucleares en la periferia de la neoformación (amplificación 10x).

muestra una típica proliferación de bandas que se fusiona del epitelio, compuesta por células pequeñas unidas por puentes y con tendencia a la formación de empalizadas periféricas. A pesar de la citología blanda, los autores mencionan como criterios de malignidad el pleomorfismo y la actividad mitósica anormal ${ }^{9}$.

El tratamiento de elección para esta patología sigue siendo la resección quirúrgica amplia con cirugía micrográfica de Mohs, cuyas tasas de curación oscilan entre el 70 y el $80 \%$. El papel de la linfadenectomía profiláctica es debatible; sin embargo, si existen ganglios o permeación intralinfática, la linfadenectomía profiláctica puede indicarse. Pese a que la pertinencia 
de la radioterapia no está bien definida, se ha reportado control de la enfermedad mediante radioterapia superficial más hipertermia. Dentro del tratamiento sistémico se han utilizado múltiples fármacos, como el metotrexato, el cisplatino, la doxorubicina y la bleomicina con resultados poco alentadores ${ }^{10}$; no obstante, hay informes de casos aislados en los que se ha administrado docetaxel como tratamiento sistémico y ha dado buenos resultados.

A nivel histológico, dentro de los factores de buen pronóstico en la revisión de patología deben ser evaluados el índice mitótico, la invasión vascular, el tamaño del tumor y la profundidad del tumor mayor de $7 \mathrm{~mm}$.

El diagnóstico diferencial debe distinguir el poroma ecrino, el carcinoma escamoso, el melanoma maligno y la metástasis cutánea ${ }^{11}$.

En nuestro caso, la lesión tumoral del paciente fue resecada completamente; por las características microscópicas se encuentra dentro del rango de los tumores de bajo grado y actualmente lleva tres años de intervalo libre sin recurrencia.

\section{Conclusiones}

Es importante realizar una adecuada valoración dermatológica a los pacientes que tienen lesiones sospechosas de cualquier lesión maligna.

Aunque la incidencia del porocarcinoma es muy baja, su comportamiento biológico se torna agresivo cuando la enfermedad se disemina y, desafortunadamente, por su baja frecuencia, continúa siendo un reto diagnóstico, así como terapéutico, ya que actualmente no existe una casuística lo suficientemente alta para determinar cuál sería el mejor tratamiento para la enfermedad localmente avanzada o avanzada. Por ello es trascendental detectar esta enfermedad cuando aún es indolente y realizar un buen tratamiento quirúrgico, así como un adecuado diagnóstico histopatológico.

\section{Aplicación práctica}

Reportamos el caso con el objetivo de aportar información acerca de las características clínicas e histológicas del tumor, así como sobre el comportamiento, incidencia, tratamiento y pronóstico de esta neoplasia infrecuente.

\section{Conflicto de intereses}

Los autores declaran no tener conflicto de intereses.

\section{Bibliografía}

1. Cruz Benítez L, Luna Rivero C, Morales Ramos R, Farias Alarcón M, Páez Valencia C, Ramírez Heredia J. Porocarcinoma ecrino. Informe de un caso y revisión de la bibliografía. GAMO. 2010;9(6):305-9.

2. Robson A, Greene J, Ansari N, Kim B, Seed PT, McKee PH, et al. Eccrine porocarcinoma (malignant eccrine poroma). A clinic pathologic study of 69 Cases. Am J Surg Pathol. 2001;25(6):710-20.

3. Lloyd MS, El-Muttardi N, Robson A. Eccrine porocarcinoma: A case report and review of the literature. Can J Plast Surg. 2003;11(3):153-6.

4. Casavilca S, Lama A, Guerrero M, Essary LR, Mantilla R, Cano L, et al. Porocarcinoma ecrino: estudio clínico-patológico de 19 casos en el Instituto Nacional de Enfermedades Neoplásicas. Rev Peru Med Exp. 2013;30(3):437-40.

5. Kurashige $Y$, Minemura T, Nagatani T. Eccrine porocarcinoma: clinical and pathological report of eight cases. Case Rep Dermatol. 2013:5:259-66.

6. Hussein K, Rasha Abo ELH. Metastatic eccrine porocarcinoma respond to combination chemotherapy docetaxel, cisplatin and infusion $5 \mathrm{FU}$ with long disease control. Am J Case Rep. 2014;3:6-11.

7. Téllez Bernal E, Fernández Tamayo NM, Trejo Rivas AA, González Rodríguez RM, Aguilar Jiménez C, Mendoza López A. Incidencia de tumores malignos en pacientes adultos diagnosticados por primera vez en el hospital Instituto de Seguridad y Servicio Social al Servicio de los Trabajadores del Estado de Puebla en el año 2013. GAMO. 2015;14(2):75-8.

8. Kim JW, Oh DJ, Kang MS, Lee D, Hwang SW, Park SW. A case of metastatic eccrine porocarcinoma. Acta Derm Venereol. 2007;87(6):550-2.

9. Calonje E, Brenn T, Lazar A, McKee P. McKee's Pathology of the Skin with clinical correlations. Fourth edition. Elsevier Saunders; 2011. pp. 1535-8.

10. Aaribi I, Mohtaram A, Ben Ameur El Youbi M, Kharmoum J, El Kabous M, Mrabti $\mathrm{H}$, et al. Successful management of metastatic eccrine porocarcinoma. Case Rep Oncol Med. 2013;2013:282536.

11. Torres Lozada V, Camacho FM, Martínez, Mihm MC, Sober A, Sánchez Carpintero I. Dermatología práctica ibero-latinoamericana: atlas, enfermedades sistémicas asociadas y terapéutica. México: Vicente Torres Lozada-Nieto Editores; 2005. 